\title{
Mudanças dos critérios Qualis!
}

\author{
Change the Qualis criteria!
}

A Associação Médica Brasileira (AMB) preocupada com o futuro das publicações científicas brasileiras, depois da divulgação dos novos critérios QUALIS da CAPES, vem desde agosto de 2009 organizando uma série de encontros em sua sede em São Paulo, em parceria com a Associação Brasileira de Editores Científicos - ABEC Brasil. Os encontros resultaram em um editorial denominado Classificação dos periódicos no sistema QUALIS da CAPES - a mudança dos critérios é URGENTE! Este foi assinado por 62 editores de revistas científicas e publicado na íntegra em todas elas e em outras inúmeras principalmente na área da saúde, evidenciando uma sensibilização e um envolvimento cada vez maior dos periódicos nacionais em discutir problemas comuns. ${ }^{1}$

A comunidade científica continua preocupada com as perspectivas, os rumos e o futuro dos periódicos brasileiros. ${ }^{2,3}$ Assim, na reunião de 18 de março os editores presentes puderam avaliar as repercussões do primeiro Editorial que serviu de base para discussões em eventos e reuniões científicas pelo país. Esta última reunião contou com a participação da Dra. Lilian Caló, Coordenadora de Comunicação Científica e Avaliação do SciElO, que apresentou estudo comparativo dos periódicos brasileiros na referida base classificados por dois critérios: o primeiro conforme o fator de impacto ISI/JCR, que usa somente as revistas indexadas na base Thomson Reuters, e segundo um índice composto pela somatória simples dos fatores de impacto ISI/JCR e do SciELO. O fator de impacto SciELO, que também considera citações de todos os periódicos da sua base, modifica significativamente o número de citações obtidas e, conseqüentemente, eleva o fator de impacto dos periódicos brasileiros. Este fato ficou mais evidente com a demonstração apresentada pela Dra. Caló do ganho percentual obtido pelos periódicos com a adoção do índice composto. Fica claro que associar outros índices, criar equivalências ou alternativas diversas podem favorecer a qualificação das revistas nacionais, melhorando sua visibilidade e favorecendo a indexação internacional. Também deve se considerar que os pesquisadores nacionais estão preferindo publicar seus conteúdos em revistas estrangeiras ao invés de fazê-los em revistas nacionais. Esta escolha, melhora a qualificação do Programa de Pós-graduação aos quais estão inseridos, conquistam fator de impacto mais elevado e aumentam o índice $\mathrm{H}$; tudo isso única $\mathrm{e}$ exclusivamente por conta dos novos critérios adotados pela CAPES. A busca de maior visibilidade $\mathrm{e}$ qualidade da produção nacional não deve ser avaliada somente pelos artigos, mas também por maior qualificação de nossos periódicos para que eles sejam reconhecidos internacionalmente.

Considerando que para atual avaliação trienal da CAPES os critérios já estão definidos, os editores reunidos decidiram elaborar um novo editorial contendo uma lista de sugestões a ser encaminhada para a coordenação da CAPES para a próxima avaliação. A lista de sugestões que complementam a do primeiro editorial é a seguinte:

- revisão dos critérios usados pela CAPES para classificação dos periódicos, sugerindo que seja adotado o fator de impacto composto pela somatória dos fatores de impacto ISI/JCR e SciELO;

- obtenção de um assento para a ABEC Brasil (Associação Brasileira dos Editores Científicos) no Conselbo Técnico Científico da CAPES, para que os editores possam ser ouvidos no processo;

- solicitação da "Bolsa do Editor" junto ao CNPq para auxílio à editoração científica destinada a editores de revistas brasileiras que recebem apoio da referida agência de fomento. Este recurso tem por objetivo aprimorar a qualidade das revistas obtendo maior dedicação de seus editores às funções editoriais;

Além disto, os editores reunidos decidiram obter apoio da Academia Brasileira de Ciências, da FINEP e do Deputado Eleuses Vieira de Paiva para suas reivindicações e sugestões. Num segundo momento os editores solicitarão ao $\mathrm{CNPq}$ detalhamento dos resultados e dos critérios adotados para distribuição dos recursos dos Editais para Auxílio à Editoração (AED). Com estas informações os editores pretendem construir um banco de dados com informações sobre orçamentos anuais dos periódicos brasileiros que será útil para análise comparativa e cooperação mútua. A divulgação dos dois editoriais e sua discussão continua sendo nossa meta em buscar o reconhecimento que os periódicos nacionais necessitam e merecem. 
Assinam este Editorial:

Adagmar Andriolo

Alfredo José Afonso Barbosa

Arnaldo José Hernandez

Aroldo F. Camargos

Benedito Barraviera

Bogdana Victoria Kadunc

Bruno Caramelli

Carlos Brites

Dejair Caitano do Nascimento

Domingo M. Braile

Dov Charles Goldenberg

Edmund Chada Baracat

Edson Marchiori

Eduardo de Paula Vieira

Eros Antônio de Almeida

Flávia Machado

Geraldo Pereira Jotz

Gianna Mastroianni Kirsztajn

Gilberto Camanho

Gustavo Gusso

Ivomar Gomes Duarte

Izelda Maria Carvalho Costa

João Ferreira de Mello Júnior

Joel Faintuch

José Antônio Baddini Martinez

José Antônio Livramento

José Eduardo Ferreira Manso

José Eulálio Cabral Filho

José Heverardo da Costa Montal

José Luiz Gomes do Amaral

José Luiz Martins

Jurandyr Moreira de Andrade

Leonardo Cançado Monteiro Savassi

Luís dos Ramos Machado

Luiz Augusto Casulari

Luiz Eugenio Garcez Leme

Luiz Felipe P. Moreira

Luiz Henrique Gebrim

Marcelo Madeira

Marcelo Riberto

Marcus Bastos

Mário Cícero Falcão

Mario J. da Conceição

Mauricio Rocha e Silva

Milton Artur Ruiz

Milton K. Shibata

Mittermayer Barreto Santiago

Nelson Adami Andreollo

Nivaldo Alonso

Osvaldo Malafaia

Olavo Pires de Camargo

Paulo Manuel Pêgo Fernandes

Regina Helena Garcia Martins

Renato Soibelmann Procianoy

Ricardo César Pinto Antunes

Ricardo Fuller

Ricardo Guilherme Viebig

Ricardo Nitrini

Rogério Dedivitis

Ronaldo Damião

Rosângela Monteiro

Sergio Lianza

Sigmar de Mello Rode

Tarcisio E.P. Barros Filho

Wallace Chamon

Winston Bonetti Yoshida

Zuher Handar
Jornal Brasileiro de Patologia e Medicina Laboratorial

Jornal Brasileiro de Patologia e Medicina Laboratorial

Revista Brasileira de Medicina do Esporte

Revista Femina

Journal of Venomous Animals and Toxins including Tropical Diseases

Surgical \& Cosmetic Dermatology da Soc. Brasileira de Dermatologia

Revista da Associação Médica Brasileira

Brazilian Journal of Infectious Diseases

Hansenologia Internationalis

Revista Brasileira de Cirurgia Cardiovascular

Revista Brasileira de Cirurgia Plástica

Revista da Associação Médica Brasileira

Revista Radiologia Brasileira

Revista Brasileira de Coloproctologia

Revista da Sociedade Brasileira de Clínica Médica

Revista Brasileira de Terapia Intensiva

Revista Brasileira de Cirurgia Cabeça e Pescoço

Jornal Brasileiro de Nefrologia

Revista Brasileira de Ortopedia

Medicina Família e Comunidade

Revista de Administração em Saúde

Anais Brasileiros de Dermatologia

Brazilian Journal of Otorhinolaryngology

Revista Brasileira de Nutrição Clínica

Jornal Brasileiro de Pneumologia

Arquivos de Neuro-Psiquiatria

Revista do Colégio Brasileiro de Cirurgiões

Revista Brasileira de Saúde Materno Infantil

Revista da Associação Brasileira de Medicina de Tráfego

Revista da Associação Médica Brasileira

Archives of Pediatric Surgery

Revista Brasileira de Ginecologia e Obstetrícia

Revista Brasileira de Medicina de Família e Comunidade

Arquivos de Neuro-Psiquiatria

Brasilia Médica

Geriatria \& Gerontologia

Arquivos Brasileiros de Cardiologia

Revista Brasileira de Mastologia

Revista Brasileira de Mastologia

Revista Acta Fisiátrica

Jornal Brasileiro de Nefrologia

Revista Brasileira de Nutrição Clínica

Revista da Sociedade Brasileira de Anestesiologia

Revista Clinics

Revista Brasileira de Hematologia e Hemoterapia

Arquivos Brasileiros de Neurocirurgia

Revista Brasileira de Reumatologia

Arquivos Brasileiros de Cirurgia Digestiva

Brazilian Journal of Craniomaxilofacial Surgery

Arquivos Brasileiros de Cirurgia Digestiva

Acta Ortopedica Brasileira

São Paulo Medical Journal

Brazilian Journal of Otorhinolaryngology

Jornal de Pediatria

Revista da Sociedade Brasileira de Cancerologia

Revista Brasileira de Reumatologia

Arquivos de Gastroenterologia

Dementia \& Neuropsychologia

Revista Brasileira de Cirurgia Cabeça e Pescoço

Urologia Contemporânea

Revista Brasileira de Cirurgia Cardiovascular

Revista Medicina de Reabilitação

Brazilian Oral Research

Acta Ortopedica Brasileira

Arquivos Brasileiros de Oftalmologia

Jornal Vascular Brasileiro

Revista Brasileira de Medicina do Trabalho 